\title{
Teaching push-down automata and Turing machines
}

\author{
César García-Osorio ${ }^{1}$, Iñigo Mediavilla-Sáiz ${ }^{1}$, Javier Jimeno-Visitación ${ }^{1}$, Nicolás García-Pedrajas ${ }^{2}$
}

\begin{abstract}
In this paper we present the new version of a tool to assist in teaching formal languages and automata theory. In the previous version the tool provided algorithms for regular expressions, finite automata and context free grammars. The new version can simulate as well push-down automata and Turing machines.
\end{abstract}

\section{Categories and Subject Descriptors}

F.4.2 [Mathematical Logic and Formal Languages]: Grammars and Other Rewriting Systems - grammar type, parsing.

\section{General Terms}

Algorithms, Languages, Theory.

\section{Keywords}

Finite automaton, regular expression, grammar, Cocke-YoungerKasami algorithm, Chomsky normal form, LL parsing, interactive teaching tool.

\section{INTRODUCTION}

One of the major problems of teaching a course on formal languages and automata theory is that the students perceive it as too theoretical, dry and difficult. Although it is possible to give a more practical approach by using examples and solving exercises, these tend to be long and tedious. In this paper we present the new functionality of "Thoth" - a tool for learning formal languages and automata (it is possible to download this tool from the following link: http://pisuerga.inf.ubu.es/cgosorio/THOTH/, or contacting the first author).

The first version was mainly focused on finite automata and regular languages and grammars, but some functionality of generating LL(1) tables for context free grammars was included as well. After the inclusion of the push-down automata and Turing machine simulation, there are few other tools that cover as many topics of the formal languages and automata field as Thoth does $[1,2,3]$. One advantage of Thoth over them is its user interface. In Thoth is possible to design a finite or push-down automaton or a Turing machine using simple mouse operations.

In the version presented here a lot of new functionality has been added that has to do with the creation and simulation of pushdown

Copyright is held by the author/owner(s).

ITiCSE'08, June 30-July 2, 2008, Madrid, Spain.

ACM 978-1-60558-115-6/08/06. automata and Turing machines. The student can design these automata models and validate interactively their functionality. Once the automata or machine has been created the student can see step by step the process of language recognition. The Turing machine model has different variation; in this version of Thoth the following have been implemented: binary, multi-tape, multihead, multi-track, tape-limited, and write/move.

Besides these new functionalities, the graphic user interface of the application has been improved. Now it is possible to undo and redo the drawing operations and the user can zoom in and zoom out the diagrams as well.

\section{CONCLUSIONS AND FUTURE WORK}

We have presented a tool that allows the fluently interaction with concepts and algorithms of automata and formal languages courses. The students avoid the tedious of doing the exercises by hand and they are free of experiment with different designs observing step by step the evolution of algorithms.

The main advantage of Thoth over other tools is its graphic user interface. It is very easy and quick to design an automaton, drawing its states and connecting them with transitions. We can avoid cluttered designs bending arcs and moving selected groups of states and arcs.

Besides, Thoth has born with the ambition of being international, the interface is prepared for four languages: Spanish, English, French and German, and the aspiration of growing up and include other kinds of devices as "Moore and Mealy machines" and "Petri nets".

In the future we expect to improve the application by means of the inclusion of other kind of automata and the possibility of using Turing machines as modules inside the design of new Turing machines.

\section{REFERENCES}

[1] J. Bovet. Visual Automata Simulator. (http://www.cs.usfca.edu/ jbovet/vas.html, last visited 4 April 2008).

[2] C. Burch. Automaton Simulator. (http://ozark.hendrix.edu/ burch/proj/autosim/, last visited 4 April 2008).

[3] S. H. Rodger. Java Interactive Formal Languages and Automata Package. (http://www.jflap.org/, last visited 4 April 2008).

\footnotetext{
${ }^{1}$ University of Burgos, Avda. Cantabría s/n, 09006, Burgos, Spain. \{cgosorio|imediava|javierjimenov\}@gmail.com.

2 University of Córdoba, Campus Universitario de Rabanales, 14071, Córdoba, Spain.npedrajas@uco.es.
} 
